\title{
The characteristics of attempted suicide by drug intoxication among emergency department visitors during 2014-2018
}

\author{
Sang Min Lee ${ }^{1}$ Minha Hong ${ }^{2}$, In Young Choi ${ }^{2}$ \\ 1. Kyung Hee University Hospital, Seoul, Korea \\ 2. Hanyang Univiersity, Myongji Hospital, Goyang, Korea \\ ${ }^{*}$ correspondence
}

Background: The drug intoxications (DI) are frequently used method in suicide attempt.

Objectives: To identify patient characteristics of drug intoxication as a suicide attempt method, and the factors associated with further psychiatric follow up.

Method: A cross-sectional study was conducted using chart review of all individuals who visited the emergency department of one university hospital following a suicide attempt during 20142018 in South Korea. (The data of 2014 were excluded owing to technical reason from system resulting in insufficient information.) The types of drug were classified into 5 categories such as prescribed psychiatric medication of oneself or family members (Prescribed PY), prescribed medications from other departments (Prescribed other), over-the-counter drugs (OTC), pesticides or rat poison (Pesticide), and combination. The primary destination of ED visitors was classified into 7 categories, which were discharge against medical advice (AMA), admission to intensive care unit (ICU), admission to general emergency department (EM-general), discharge due to recovery $(D / C)$, admission to psychiatric department, and other. (3 cases were transferred to other hospital due to lack of admission beds.) After discharge from the hospital, PY outpatient visits of each patient were tracked through medical record.

Results: Among the visitors of ED, total of 474 was suicide attempters by drug intoxication (male 34.3\%, female 65.7\%). The number of suicide attempters by drug intoxication was increasing during study period (96 in 2015, 112 in 2016, 118 in 2017, 151 in 2018). The age and gender distribution were shown in Figure 1. The number of female attempters via DI were dominant in all age groups, and the peak age group was age 50-59 in both male and female.
The types of drug in each age group were shown in Figure 2. The prescribed psychiatric medication of oneself or family members were the highest in almost all age groups except age 70 and over. The PY outpatient follow ups after primary destination were depicted in Figure 3. Except for PY admitted patients, the majority of the patients did not visit PY outpatient clinic after discharge.

Conclusions: It is important to consult or connect with psychiatric department in the management of suicide attempts by drug intoxication.

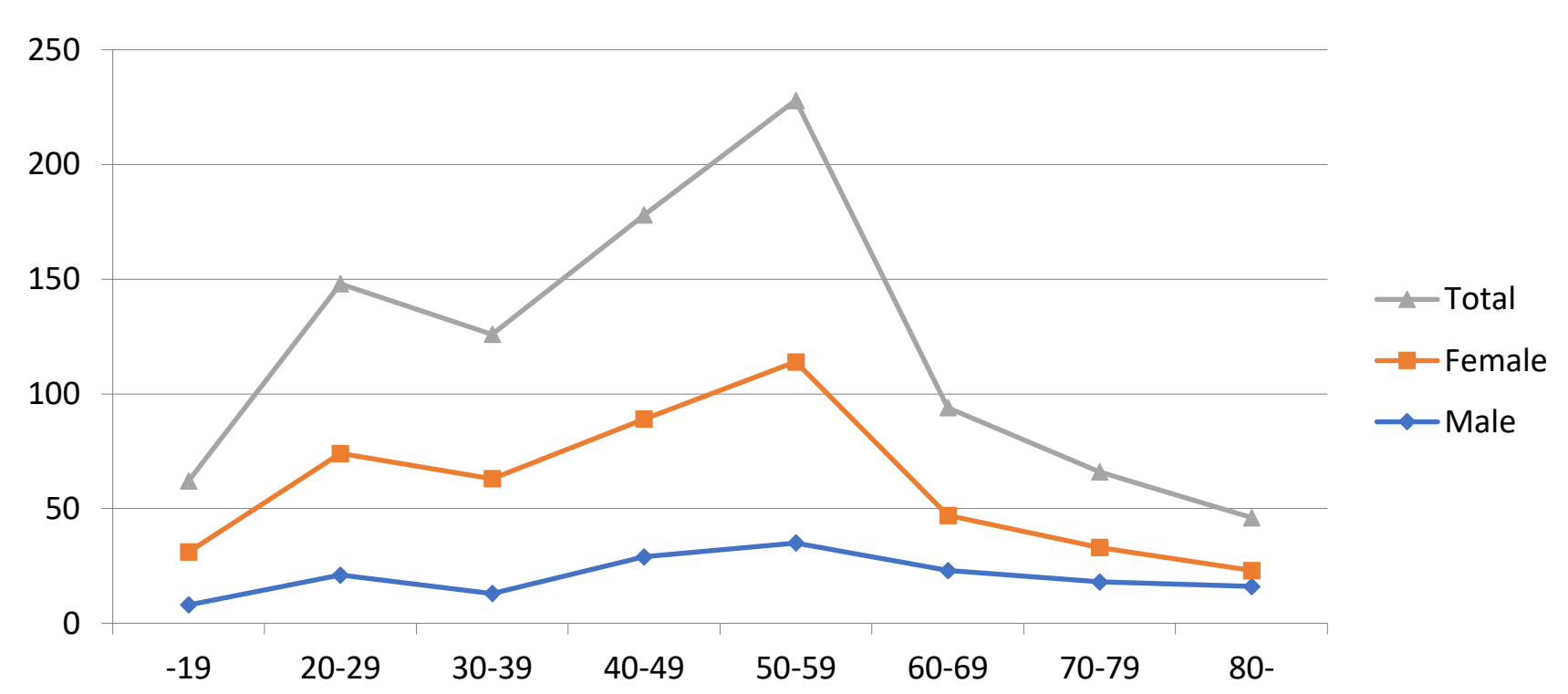

Figure 1. The number of DI in each age group

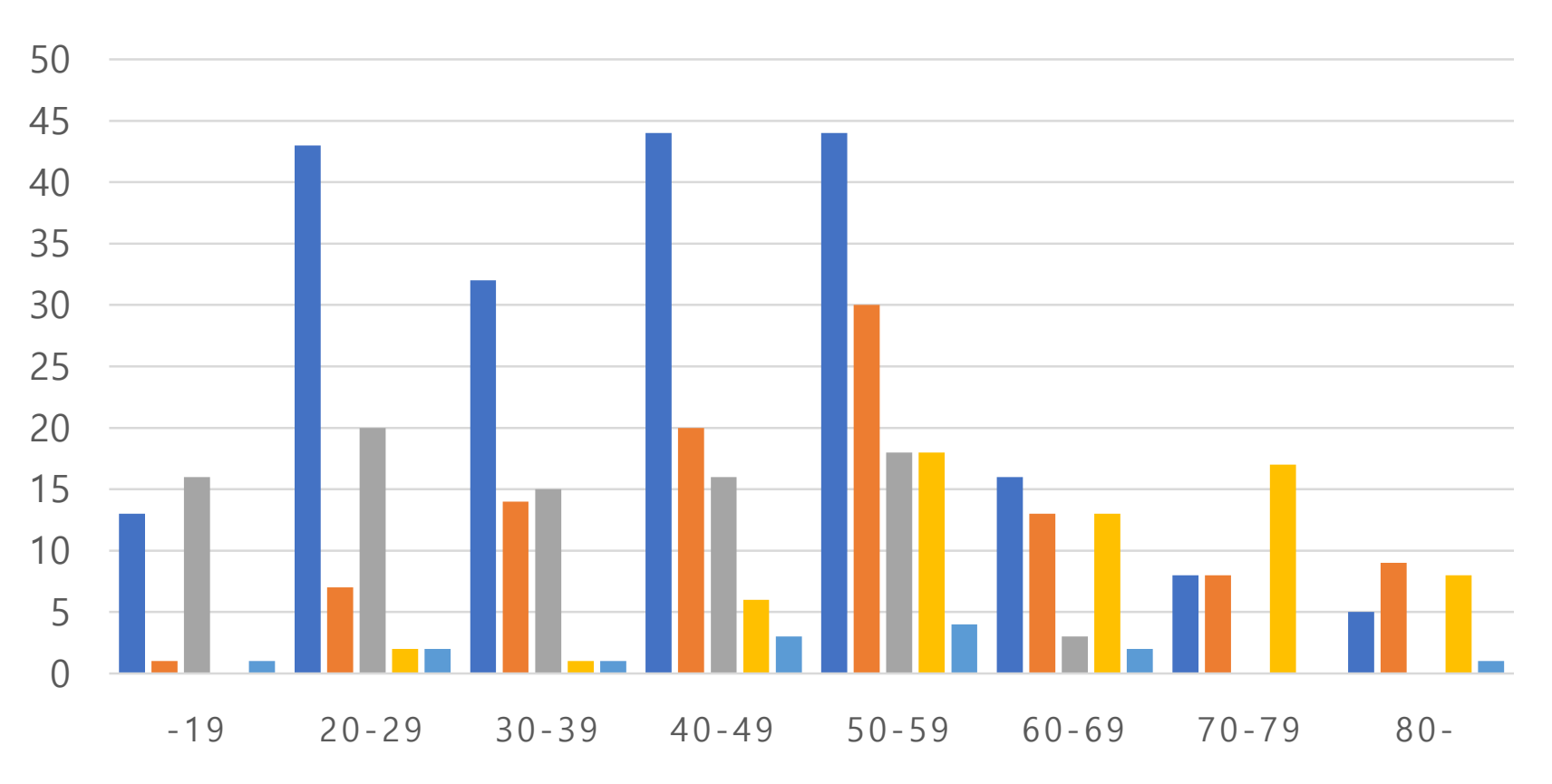

- Prescribed PY $\backsim$ Prescribed Other $\square$ OTC drug $\square$ Pesticides $\square$ Combination Figure 2. The types of drug in each age group

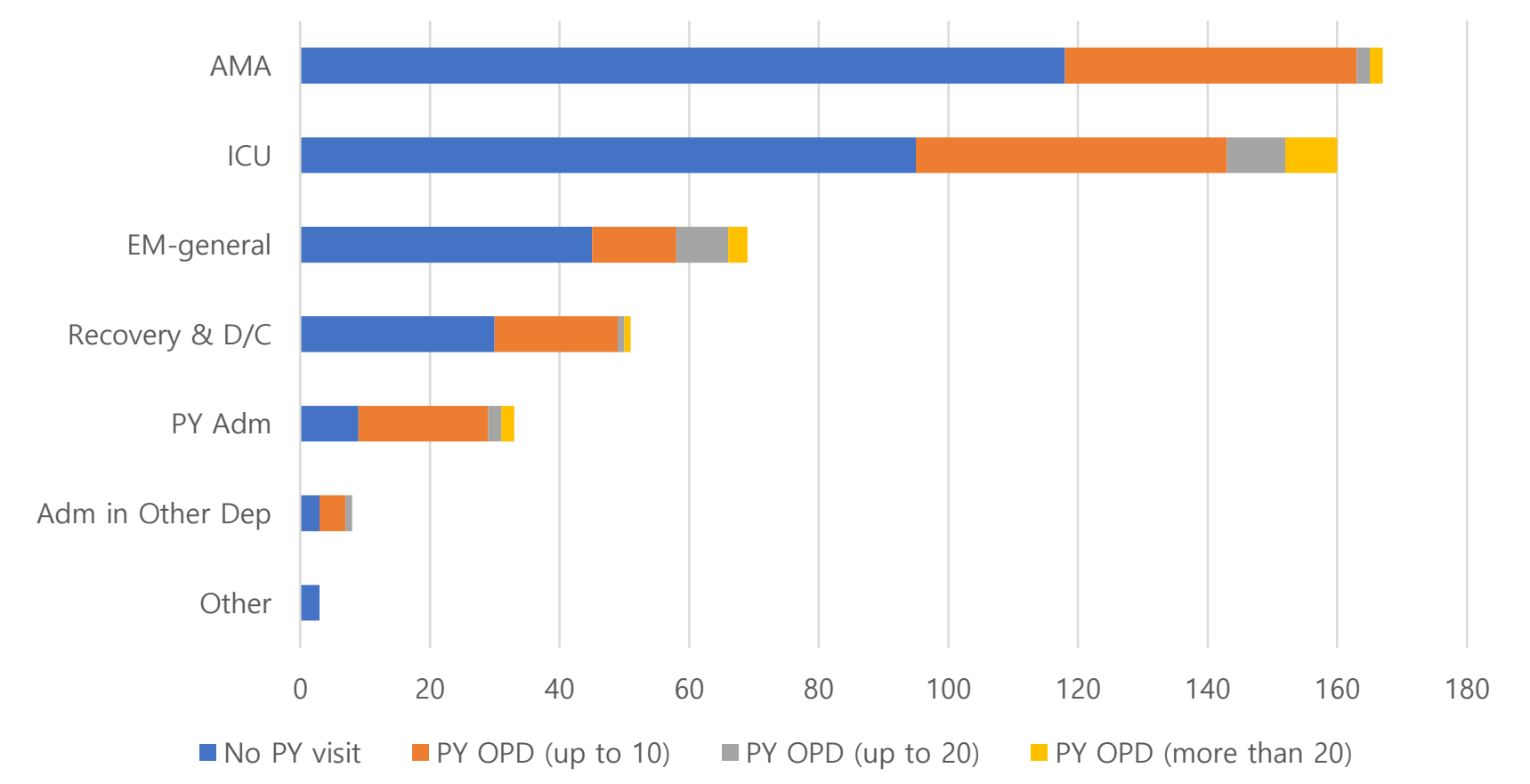

Figure 3. The PY OPD follow-ups after discharge 